\title{
Automatic Tuning of Data Distribution Using Factoring in Master/Worker Applications ${ }^{1}$
}

\author{
Anna Morajko, Paola Caymes, Tomàs Margalef, and Emilio Luque \\ Computer Science Department. Universitat Autònoma de Barcelona, \\ 08193 Bellaterra, Spain \\ \{ania, paola\} @aomail.uab.es \\ \{tomas.margalef, emilio.luque\}@uab.es
}

\begin{abstract}
Parallel/Distributed programming is a complex task that requires a high degree of expertise to fulfill the expectations of high performance computation. On the one hand, application developers must tackle new programming paradigms, languages, libraries. On the other hand they must consider all the issues concerning application performance. On this context the Master/Worker paradigm appears as one of the most commonly used because it is quite easy to understand and there is a wide range of applications that match this paradigm. However, to reach high performance indeces it is necessary to tune the data distribution or the number of Workers considering the particular features of each run or even the actual behavior that can change dynamically during the execution. Dynamic tuning becomes a necessary and promising approach to reach the desired indeces. In this paper, we show the usage of a dynamic tuning environment that allows for adapting the data distribution applying Factoring algorithm on Master/Worker applications. The results show that such approach improves the execution time significantly when the application modifies its behavior during its execution.
\end{abstract}

\section{Introduction}

Parallel and distributed systems offer high computing capabilities that are used in many scientific research fields. These capabilities have taken the evolution of science to a new step called computational science. This facilitated the determining of the human genome, computing the atomic interactions in a molecule or simulating the evolution of the universe. So biologists, chemists, physicians and many other researchers have become intensive users of high performance computing. They submit very large applications to powerful systems in order to get the results as fast as possible, considering the largest problem size and taking advantage of the resources available in the system. The increasing need of high performance systems has driven scientists towards parallel/distributed systems. Although such systems have their performance limits, they are much more powerful than the rest of the computers and hence are better for solving scientific problems demanding intensive computation.

1 This work has been supported by the MCyT (Spain) under contract TIC2001-2592 and has been partially supported by the Generalitat de Catalunya - GRC 2001SGR-00218. 
Problems covered by computational science usually comprise a lot of calculations which are easily parallelizable, and consequently applications to solve these problems can be designed using the Master/Worker (M/W) paradigm [1]. Typically, in the M/W paradigm a Master process in each iteration distributes data among Worker processes and waits for their response. Each Worker processes its portion of data independently of the remaining Workers and returns the results to the Master. When the Master receives results from the Workers, it may distribute the data again. There are many cases the Master must synchronize all the results from all the Workers before the next data distribution. However, heterogeneous computing and communication powers, or varying the amount of distributed data could cause load imbalance. In these situations slower or overloaded machines and/or incorrect data distribution may significantly increase the idle time of processes and influence into the application execution time. The workload balancing goal therefore is to minimize the idle time and calculate the right amount of data for each process. Load balancing should be achieved in such a way that fast computers will automatically process more amount of data than the slower ones. Moreover, an optimal data distribution may also depend on dynamic factors such as input data, network load, computer load and so on. Before the application execution, developers do not know these parameters, hence they cannot distribute the work properly. Therefore, it can be beneficial to dynamically tune the data distribution by adapting it to changing conditions. In this context, our goal is to balance and distribute data correctly and dynamically among the available processes taking into account capabilities and load of the machines the application runs on.

In the following sections of this paper, we present a complete performance optimization scenario that considers the data distribution problem in a dynamic approach. In Section 2, we describe the algorithm used to distribute the data. In Section 3, we analyze the tuning of the data distribution by using the MATE environment that supports dynamic tuning of parallel applications. In Section 4, we present the results of experiments conducted in the MATE to dynamically tune the data distribution using the presented algorithm. Finally, Section 5 shows the conclusions of this work.

\section{Factoring Data Distribution}

The data distribution from a Master process to the Worker ones can be done in several different ways considering different algorithms. One possibility is to divide the total data $n$ in $p$ chunks, where $\mathrm{p}$ is the number of Workers and distribute one of these chunks to each Worker. However, if there is any heterogeneity in the system the faster processors will be waiting for the slower ones. Another possibility is to divide the total data in $\mathrm{m}$ same-size chunks, where $\mathrm{m}$ is greater than $\mathrm{p}$. In this case, the Master distribute the $\mathrm{p}$ first chunks to the Workers. When one Worker finishes its work, it returns the result to the Master and if there are chunks to be processed, the Master sends to that Worker a new chunk. A third possibility is to create chunks of different size in such a way that the initial distribution consider bigger chunks and when there are less data to be processed, the amount distributed is smaller.

One of the algorithms using this third approach is the Factoring Scheduling algorithm [2]. In the factoring algorithm, the work is partitioned according to a factor into a set of different-size tuples. The Master distributes one tuple to each Worker. If a 
Table 1. Examples of tuple sizes for different factors

\begin{tabular}{|l|l|l|l|l|}
\hline $\begin{array}{l}\text { Work } \\
\text { size }(\mathrm{N})\end{array}$ & $\begin{array}{l}\text { Number of } \\
\text { Workers } \\
(\mathrm{P})\end{array}$ & $\begin{array}{l}\text { Factor } \\
(\mathrm{f})\end{array}$ & $\begin{array}{l}\text { Threshold } \\
(\mathrm{T})\end{array}$ & Tuples \\
\hline 1000 & 2 & 1 & 1 & 500,500 \\
\hline 1000 & 2 & 0.5 & 1 & $\begin{array}{l}250,250,125,125,63,63,32,32,16,16,8,8,4,4, \\
2,2,1,1\end{array}$ \\
\hline 1000 & 2 & 0.5 & 16 & $\begin{array}{l}250,250,125,125,62,62,32,32,16,16, \\
16,16\end{array}$ \\
\hline 1000 & 2 & 0.7 & 1 & $350,350,105,105,31,31,10,10,3,3,1,1$ \\
\hline 1000 & 4 & 1 & 1 & $250,250,250,250$ \\
\hline 1000 & 4 & 0.5 & 1 & $\begin{array}{l}125,125,125,125,62,62,62,62,32,32, \\
32,32,16,16,16,16,8,8,8,8,4,4,4,4,2, \\
2,2,2,1,1,1,1\end{array}$ \\
& & & & $\begin{array}{l}125,125,125,125,62,62,62,62,32,32, \\
32,32,16,16,16,16,16,16,16,16\end{array}$ \\
\hline 1000 & 4 & 0.5 & 16 & $\begin{array}{l}175,175,175,175,52,52,52,52,16,16, \\
16,16,5,5,5,5,1,1,1,1,1,1,1,1,\end{array}$ \\
\hline 1000 & 4 & 0.7 & 1 &
\end{tabular}

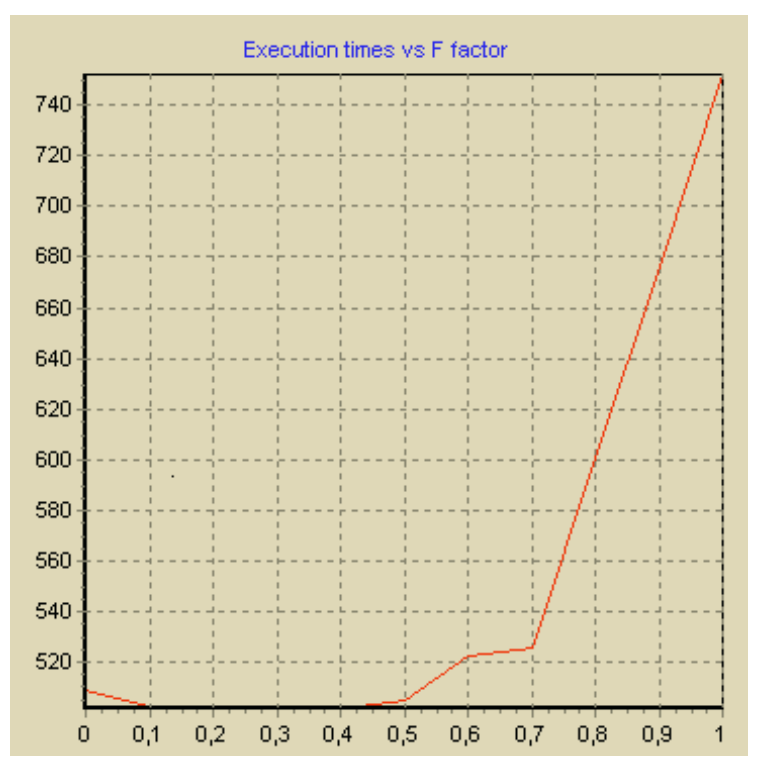

Fig. 1. Execution time in function of factor $\mathrm{f}(\mathrm{N}=1000, \mathrm{P}=4, \mathrm{~T}=1$, relative speeds: $1,3,3,3)$

Worker has finished its tuple and there are tuples to be processed, then it receives the next tuple. This cycle is repeated till all the tuples of the work are processed.

Assuming there are $\mathrm{P}$ parallel Workers, a threshold $\mathrm{T}>0$ (minimal tuple size) and a factoring value $(0<\mathrm{f}<=1)$, the sizes of factored work tuples are calculated according to the following algorithm [2]: 

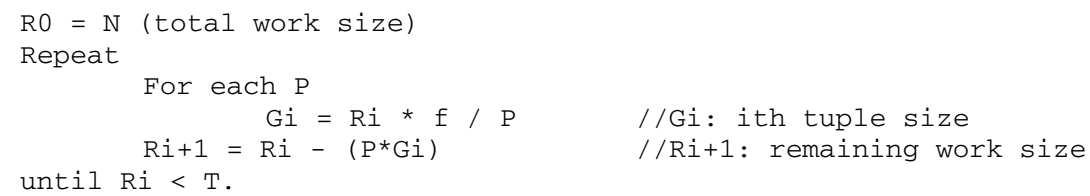

The example tuple sizes calculated according to the presented above factoring algorithm are shown in Table 1.

Figure 1 shows the execution time of an example application in a particular condition considering different values for the factor $\mathrm{f}$. As it can be observed, the value of the factor $\mathrm{f}$ influences strongly the execution time of the application.

\section{Tuning Factor $f$ with MATE}

To provide dynamic automatic tuning of parallel/distributed applications we have developed an environment called MATE (Monitoring, Analysis and Tuning Environment) [3]. MATE performs dynamic tuning in three basic and continuous phases: monitoring, performance analysis and modifications. This environment dynamically and automatically instruments a running application to gather information about the application behavior. The analysis phase receives events, searches for bottlenecks, detects their causes and gives solutions on how to overcome them. Finally, the application is dynamically tuned by applying given solution. Moreover, while it is being tuned, the application does not need to be re-compiled, re-linked and restarted.

The knowledge to represent the performance model of each particular performance problem is specified in a component called "tunlet". Each tunlet includes the information concerning the measure points to insert instrumentation in the target application, the performance model to determine the behavior of the application and the required modifications, and the tuning actions to improve the application performance.

We have been conducted many various practical experiments on parallel/distributed applications to see if our approach really works. We have proven that it is effective, profitable, and can be used for a real improvement of the program performance. Running applications under control of MATE has allowed for adapting their behavior to the existing conditions and improving their functionality [4].

To dynamically tune the data distribution we had to consider several issues:

- How to calculate work tuples (factoring algorithm described in previous section).

- How to distribute the data according to the given factor.

- How to calculate the optimal Factor and what is required to calculate this value.

To tune the Factor $\mathrm{f}$ applying the MATE environment, the application must be prepared for the tuning actions and hence it must implement adequately the data distribution: 


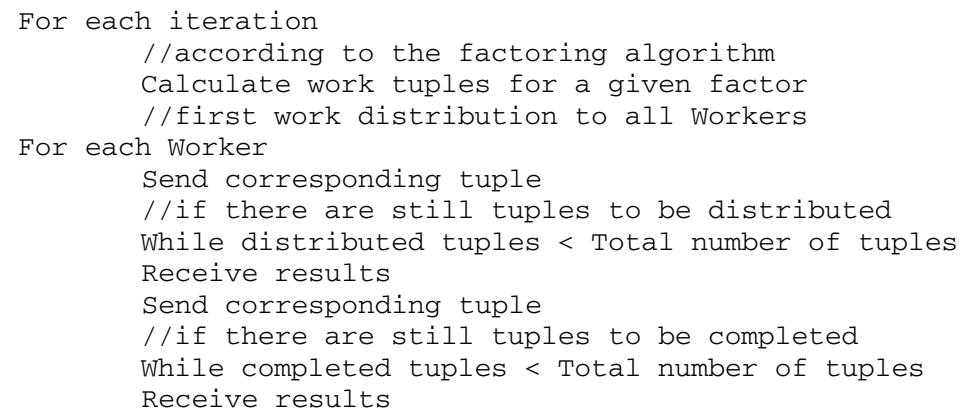

During the execution, we can monitor the PVM functions responsible for exchanging messages, e.g. pvm_send() and pvm_recv(). In particular, by monitoring: send entry/exit, receive entry/exit events in the Master process, and receive entry/exit and send entry/exit in all Worker processes, we are able to perform all necessary measurements. To find the optimal value of the Factor the performance model must provide the prediction of the iteration time. For this purpose we implemented the simulation of the application iteration time according to the following:

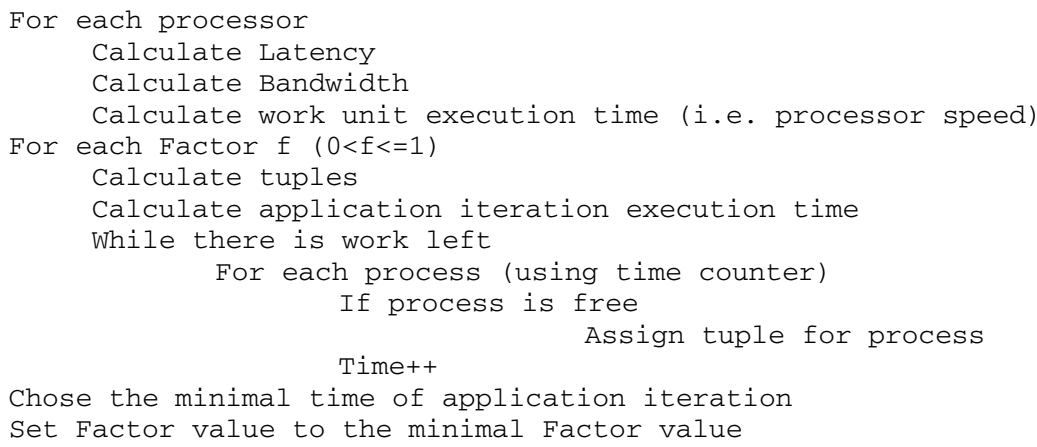

The presented algorithm simulates the execution of the program iteration for each chosen possible value of Factor $\mathrm{f}$. The simulation uses basic measurements such as network latency, bandwidth and current speed for each processor. The algorithm simulates the complete iteration by assigning subsequent work tuples to next free processor. It takes into account the time for sending the work, time for processing the work on the selected processor in function of its current speed (i.e. capacity and current load), and time for returning the result. The iteration finishes when all tuples have been processed, and the simulation records its execution time. After simulating iterations for all chosen factors, the algorithm returns the value $\mathrm{f}$ for the iteration with the shortest execution time.

Considering the algorithm presented above we must receive a set of metrics to calculate the optimal Factor such as, network latency, network bandwidth, average Worker speed expressed as the work unit processing time. To simplify the implementation, we have assumed network latency to be $1 \mathrm{~ms}$. Network bandwidth can be computed using the following formulas: 


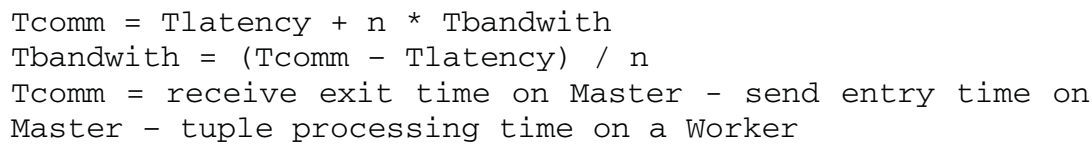

Work unit processing time is computed as the averaged value of the tuple processing time divided by number of work units per tuple. This is calculated individually for each Worker. The average is taken for all work units processed during last iteration. This assumes the total iteration time is not very large and the average can express current machine load. If the iteration time is long, this should be changed to an average taken for a given time window. Each iteration updates the work unit processing time value for each Worker and in this way we can keep track of dynamic variations in the load of the machines. It must be pointed out that this approach is suitable only if the work unit processing time depends on the work unit size and not on its content.

If a new value of the Factor is different than the current one, then the tuning action should be performed. The tuning action includes the modification of the value of specific predefined application variable. This variable represents the Factor of the work and its name is well known to the tunlet. When a next iteration is performed in the application, first, the new value of the Factor should be applied to recalculate the work tuples, and then the data can be distributed. The variable modification does not need to be synchronized, as the application will use its value next time the iteration of work processing starts.

\section{Experimental Results}

In this section, the experimental results obtained by applying the tuning environment to a real Master/Worker application are presented.

To conduct the experiments, we selected an intensive computing Forest Fire Propagation application called Xfire [5]. The Xfire application is a Master/Worker PVM based implementation of the simulation of the fireline propagation. It calculates the next position of the fireline considering the current fireline position and different aspects as weather, wind, vegetation, etc.

In every scenario, one Worker was executed in the same machine as Master. Remainder Workers were executed in individuals machines.

Experiments were conducted on a cluster of homogenous Pentium 4, $1.8 \mathrm{Ghz}$, (SuSE Linux 8.0) connected by $100 \mathrm{Mb} / \mathrm{sec}$ network. Each experiment was performed three times and the average of the execution time for the application was calculated.

We have conducted our experiments in three scenarios:

- In the first scenario, Xfire was executed in dedicated machines without any tuning and it was used as a reference result.

- In the second scenario, Xfire was executed in a non-dedicated environment; in this case we have introduced a controlled variable load, using our Load Generator tool. We generated $75 \%$ CPU load in a half of machines every eight iterations. 
- The third scenario is exactly the same on the third one, but in this case Xfire was executed under MATE applying the tuning of the data distribution. In this way, we demanded more adaptability to the load balancing.

Table 2 summarizes the experimental results. These results are also presented in figure 2 .

Table 2. Execution time of Xfire (in seconds) considering different number of Workers

\begin{tabular}{|l|c|c|c|c|c|c|}
\hline Scenario & $\mathbf{1}$ & $\mathbf{2}$ & $\mathbf{4}$ & $\mathbf{8}$ & $\mathbf{1 6}$ & $\mathbf{3 0}$ \\
\hline Xfire & 17663 & 9126 & 4732 & 2488 & 1350 & 880 \\
\hline Xfire + variable load & & 11906 & 6098 & 3157 & 1501 & 1208 \\
\hline Xfire + MATE + variable load & & 10206 & 5684 & 2784 & 1487 & 1073 \\
\hline
\end{tabular}

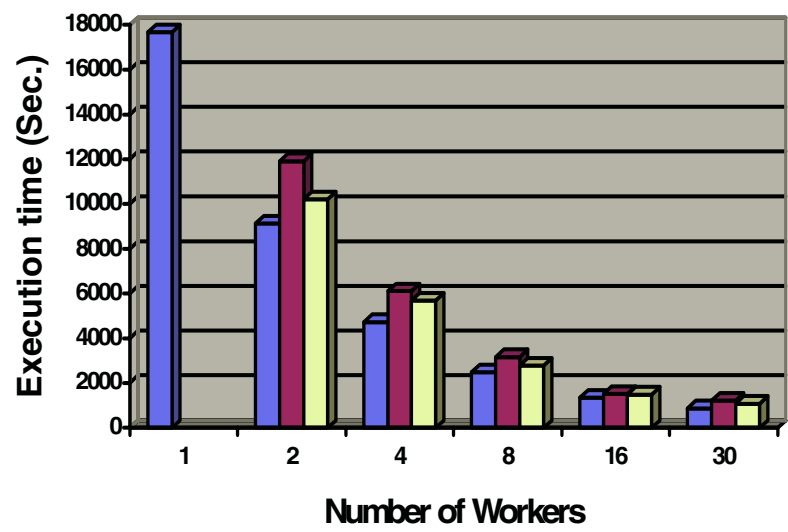

\begin{tabular}{|l|}
\hline XXfire \\
पExtra Load \\
पMATE
\end{tabular}

Fig. 2. Execution time of Xfire considering different number of Workers

This figure shows the execution time of the Xfire application considering different number of Workers, the execution time of Xfire with additional load and the execution time of Xfire with additional load under the control of MATE. It can be observed that the introduction of an extra load provokes an increase in the execution time of the application. This is due to the new extra load, but also to the load imbalance introduced by the wrong Factor distribution in the new situation. The execution under MATE control is able to detect the load imbalance and correct Factor to improve the execution time.

\section{Conclusions}

Parallel and distributed programming offers high computing capabilities to the users in many scientific research fields. The performance of applications written for such environments is one of the crucial issues. Data distribution appears as one of the most significant problems in Master/Worker applications. There are several approaches to 
distribute data, but the main goal is to accomplish that there are no Workers idle waiting for busy ones. One of this approaches is the Factoring Scheduling. However, the factoring requires the tuning of the Factor $f$ that can change dynamically depending on system load. In this context we show the applicability of the MATE dynamic tuning environment to improve the data distribution and the application performance by tuning the value of the Factor $f$. The experimental results show that the MATE environment is very useful and improves the execution time significantly adapting application to changing conditions.

\section{References}

1. César, E., Mesa, J.G., Sorribes, J., Luque, E. "Modeling Master-Worker Applications in POETRIES”. IEEE $9^{\text {th }}$ International Workshop HIPS 2004, IPDPS, pp. 22-30. April, 2004.

2. Hummel, S.F., Schonberg, E., Flynn, L.E., "Factoring: A method for Scheduling Parallel Loops", Communications of the ACM, 35, no 8, August 1992.

3. Morajko, A., Morajko, O., Jorba, J., Margalef, T., Luque, E. "Dynamic Performance Tuning of Distributed Programming Libraries". LNCS, 2660, pp. 191-200. 2003.

4. Morajko, A., Morajko, O., Margalef, T., Luque, E.. "MATE: Dynamic Performance Tuning Environment". LNCS, 3149, pp. 98-107 (2004).

5. Jorba, J., Margalef, T., Luque, E., Andre, J, Viegas, D.X. "Application of Parallel Computing to the Simulation of Forest Fire Propagation", Proc. 3rd International Conference in Forest Fire Propagation, Vol. 1, pp. 891-900. Portugal, November 1998. 